\title{
NOUVELLE
}

\section{Un peu de Rose dans la maladie de Parkinson}

Arnaud Berthier
Centre de Recherche Principe Felipe, Laboratoire 1-01, Av Autopista del Saler, 16-3, 46013 Valencia, Espagne. aberthier@yahoo.fr
> La plupart d'entre nous considère la maladie de Parkinson (MP) comme une maladie du troisième âge puisque ses symptômes apparaissent le plus souvent chez les personnes âgées de plus de 65 ans. Cependant, les formes familiales peuvent être plus précoces (âge de début avant 40 ans), ou être même juvéniles (âge de début avant 20 ans) et leur identification a permis d'énormes progrès dans la compréhension de sa physiopathologie. Ainsi, depuis la découverte en 1997 de la première forme héréditaire de la MP qui a pu être associée à une mutation d'un seul gène, SNCA ( $\alpha$-synucléine), cinq gènes sont à ce jour connus pour provoquer (sans ambiguïté) des formes familiales de maladie de Parkinson (SNCA, parkine, DJ-1, PINK1-Pten-Induced Kinase 1, ATP13A2, LRKK2) [1,2]. Ces découvertes ont apporté, au niveau moléculaire, plusieurs indices pour mieux comprendre l'étiologie de cette maladie. En effet, bien que de telles mutations ne soient observées que dans une minorité des cas, il est raisonnable de penser que des mécanismes similaires interviennent dans les formes héréditaires et sporadiques de la MP.

Même si une voie commune reliant tous les gènes associés à la MP n'a pas encore été mise en évidence, il apparaît que la mitochondrie joue probablement un rôle central. Diverses études ont ainsi montré que des toxines touchant la mitochondrie induisaient la MP et que l'activité du complexe I mitochondrial était réduite chez les patients atteints de cette maladie [3]. Enfin, au moins quatre gènes associés à des formes familiales sont impliqués dans le fonctionnement de la mitochondrie.

Ainsi, il a été démontré que les protéines Parkine et DJ-1, qui sont essentiellement cytoplasmiques, pouvaient également être localisées dans la mitochondrie [4, 5]. De plus, des études in vivo ont montré que des mutations du gène murin parkine ainsi que de son homologue chez Drosophila melanogaster induisent, chez ces animaux, des imperfections structurales des mitochondries ainsi que des défaillances énergétiques $[6,7]$.

Les découvertes récentes, chez des patients souffrant de formes héréditaires de la MP, de mutations dans les gènes de PINKI et de Omi/HtrA2 (high temperature requirement protein $A 2$ ) associent encore plus directement la mitochondrie à cette maladie (Figure 1) [8].

La protéine PINKl est une protéine de 581 acides aminés qui contient un domaine sérine/thréonine kinase, ainsi qu'un peptide/signal/message d'adressage vers la mitochondrie. Cette dernière région est clivée lors de l'insertion de PINKl dans la membrane interne mitochondriale, exposant ainsi son domaine kinase dans l'espace intermembranaire mitochondrial. La protéine 0mi/HtrA2 est, quant à elle, une sérine protéase tout d'abord identifiée comme étant I'homologue chez les mammifères des endopeptidases bactériennes HtrA (DegP) et DegS. Tout comme PINKl, $0 \mathrm{mi} / \mathrm{HtrA} 2$ contient une séquence aminoterminale de localisation mitochondriale. Durant l'apoptose, Omi/HtrA2 est libérée de la mitochondrie et se localise dans le cytosol, où elle s'unit aux protéines inhibitrices de l'apoptose (IAP). Cette liaison permet, d'une part, de contrecarrer les effets inhibiteurs des IAP vis-à-vis des caspases, et, d'autre part, d'augmenter l'activité protéolytique de 0mi/HtrA2 [8].
Bien que les souris $P I N K 1^{-/-}$ne présentent aucune imperfection mitochondriale, des mutations de l'homologue de PINKI chez Drosophila melanogaster altèrent leurs mitochondries, particulièrement dans les cellules musculaires et génitales. Ces mouches sont stériles, incapables de voler, et meurent prématurément. Ces différentes anomalies, similaires à celles qui sont observées chez les mouches ayant des mutations dans le gène homologue de Parkine, sont abolies par la surexpression de PINKl ou de Parkine. $\varepsilon$ n revanche, seule la surexpression de Parkine restaure un phénotype normal chez les mouches déficientes en Parkine. Ces observations suggèrent que PINKl et Parkine partagent une même voie de signalisation cellulaire, et que PINKl agirait en amont de Parkine [9].

Une étude récente réalisée par Pridgeon et al. permet de mieux comprendre la fonction de PINKl [10]. En effet, ces auteurs ont démontré que la protéine TRAPI (TNF receptor-associated protein 1 ), une protéine chaperon présente dans l'espace intermembranaire mitochondrial, était un partenaire moléculaire de PINK1. Lorsque PINKl est surexprimée, la phosphorylation de TRAPl est exacerbée, tout comme lors de la réponse cellulaire à un stress oxydant. De plus, la surexpression de la forme normale de PINKl, et non celle de ses formes mutées présentes dans la MP, protège la cellule de l'apoptose sauf lorsque l'expression de TRAPl est inhibée. Pris dans leur ensemble, ces résultats montrent que PINKl agit juste en amont de TRAPl dans une même voie de signalisation anti-apoptotique.

Une autre voie intéressante dans la compréhension de la fonction de PINKl est 
celle découverte par Plun-Favreau et al. [11]. Ces auteurs ont en effet montré que la protéine $0 \mathrm{mi} / \mathrm{HtrA} 2$ interagissait avec PINKl, et que ces deux protéines faisaient partie d'une même voie de signalisation cellulaire. Ainsi, ces auteurs ont démontré, d'une part, grâce à l'utilisation d'ARN interférents, que PINKl induisait la phosphorylation de 0mi/Htra2 sur des résidus sérines proches d'acides aminés mutés chez des patients atteints de la MP, et, d'autre part, que la phosphorylation de $0 \mathrm{mi} / \mathrm{HtrA} 2$ était très largement atténuée chez des patients atteints de la MP induite par des mutations de PINKI. La phosphorylation de $0 \mathrm{mi} / \mathrm{HtrA} 2$ diminue son activité protéolytique, et de façon surprenante, protège la cellule de stress impliquant la mitochondrie. Les auteurs suggèrent alors que $0 \mathrm{mi} / \mathrm{HtrA} 2$ fonctionnerait plus comme ses homologues bactériens en dégradant des protéines mal repliées, en contrôlant la fonction des protéines mitochondriales, ou encore en participant à la transduction de la réponse adaptative à des signaux de stress.

Cependant, ces études posent plusieurs questions: tout d'abord, Plun-Favreau et al. suggèrent que PINKl participerait à la phosphorylation anti-apoptotique de 0mi/HtrA2 induite par l'activation de la voie p38 MAPK. Toutefois, ils ne montrent pas que PINKl phosphoryle directement Omi/HtrA2, ni comment la phosphorylation de 0mi/HtrA2 inhibe l'apoptose. De même, Pridgeon et al. ont réalisé leur étude dans des lignées cellulaires, et il reste à vérifier que la voie PINKl-TRAPl agit également dans des neurones primaires et plus particulièrement dans le contexte de la MP. De plus, les mécanismes exacts par lesquels TRAPl inhibent l'apoptose restent encore à élucider. Par ailleurs, pourrait-il exister un lien fonctionnel entre $0 \mathrm{mi} / \mathrm{HtrA} 2$ et TRAPl,

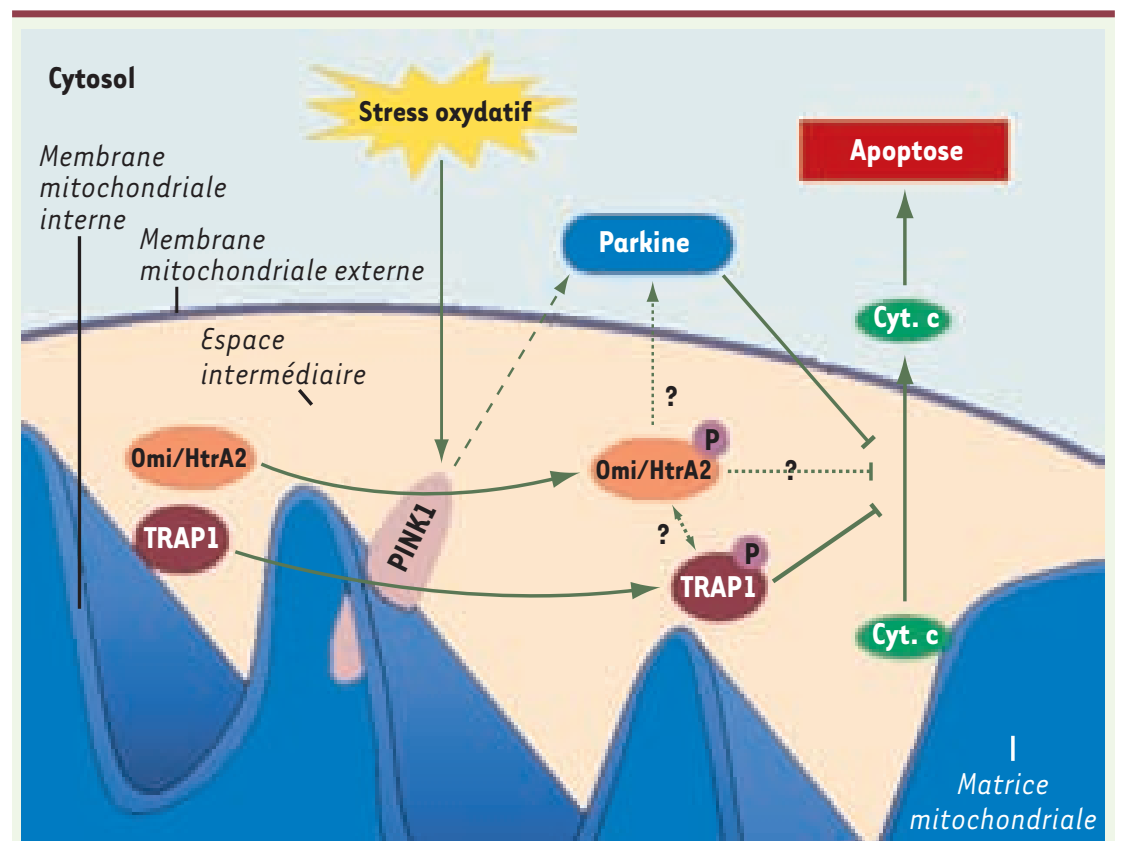

Figure 1. Rôle de PINKI dans la maladie de Parkinson. La mort cellulaire par apoptose associée à un stress oxydatif, comme observé dans la maladie de Parkinson, est inhibée par la protéine kinase PINK1. D'une part, en phosphorylant la protéine chaperon TRAPI, PINKI inhiberait la libération dans le cytosol du cytochrome c, où il participe à l'induction de l'apoptose. D'autre part, PINK1 induirait la phosphorylation de la protéase 0mi/HtrA2 inhibant alors l'apoptose. Enfin, la protéine Parkine, qui est impliquée elle aussi dans des formes héréditaires de la maladie de Parkinson, inhibe également l'apoptose. Cependant, la (ou les) voie(s) par la(es)quelle(s) elle exerce son action anti-apoptotique sont encore inconnues, et on ne sait pas par exemple si PINKI et Parkine peuvent interagir directement ou via des intermédiaires. Cyt. c : cytochrome $c$. c'est-à-dire interviennent-elles dans la même voie anti-apoptotique? D'un autre coté, PINKl agit-elle uniquement en phosphorylant TRAPl et $0 \mathrm{mi} / \mathrm{HtrA2}$, ou peutelle être impliquée dans d'autres fonctions comme dans le maintien de l'homéostasie calcique ou encore dans la régulation de la phosphorylation oxydative, deux voies importantes dans la survie neuronale? Enfin, une dernière question cruciale serait de connaître la place de la Parkine dans ces voies de signalisation cellulaire.

Pour conclure, en impliquant PINKl dans des mécanismes anti-apoptotiques au niveau mitochondrial, les travaux de Plun-Favreau et al., et de Pridgeon et al., montrent que PINKl joue un rôle essentiel dans la MP et pourrait ainsi se révéler une cible potentielle dans le traitement de celle-ci. $\diamond$

\section{A little bit of PINK}

in Parkinson's disease

\section{RÉFÉRENCES}

1. Polymeropoulos MH, Lavedan C, Leroy $\varepsilon$, et al. Mutation in the alpha-synuclein gene identified in families with Parkinson's disease. Science 1997; 276: 2045-7.

2. Corti 0, Brice A. La maladie de Parkinson: que nous apprennent les gènes responsables des formes familiales? Med Sci (Paris) 2003 ; 19 : 613-9.

3. Beal MF. Mitochondria, oxidative damage, and inflammation in Parkinson's disease. Ann Ny Acad Sci 2003 ; 991 : 120-31.

4. Darios F, Corti 0 , Lucking CB, et al. Parkin prevents mitochondrial swelling and cytochrome $\mathrm{c}$ release in mitochondria-dependent cell death. Hum Mol Genet $2003 ; 12: 517-26$

5. Zhang L, Shimoji M, Thomas B, et al. Mitochondrial localization of the Parkinson's disease related protein DJ-1: implications for pathogenesis. Hum Mol Genet $2005 ; 14: 2063-73$.

6. Palacino JJ, Sagi D, Goldberg MS, et al. Mitochondrial dysfunction and oxidative damage in parkin-deficient mice. J Biol Chem 2004 ; 279 : 18614-22.

7. Greene JC, Whitworth AJ, Kuo I, et al. Mitochondrial pathology and apoptotic muscle degeneration in Drosophila parkin mutants. Proc Natl Acad Sci USA $2003 ; 100: 4078-83$.

8. Klein C, Schlossmacher M. The genetics of Parkinson's disease. Nat Clin Pract Neurol 2006 ; 2 : 136-46.

9. Clark IE, Dodson MW, Jiang C, et al. Drosophila pink1 is required for mitochondrial function and interacts genetically with parkin. Nature $2006 ; 441$ : 1162-6.

10. Pridgeon JW, Olzmann JA, Chin LS, et al. PINK1 protects against oxidative stress by phosphorylating mitochondrial chaperone TRAPI. PLOS Biol 2007 ; 5 : el72.

11. Plun-Favreau H, Klupsch K, Moisoi N, et al. The mitochondrial protease $\mathrm{HtrA2}$ is regulated by Parkinson's disease-associated kinase PINK1. Nat Cell Biol 2007 ; 9 : 1243-52. 